\title{
Neurological involvement associated with plasmodium vivax malaria from Pakistan
}

\author{
Yousaf Abdullah Khan \\ Aga Khan University \\ Usman Hameed Mian \\ Aga Khan University \\ Najia Karim Ghanchi \\ Aga Khan University, najia.ghanchi@aku.edu \\ Ali Bin Sarwar Zubairi \\ Aga Khan University, ali.zubairi@aku.edu \\ Mohammad Asim Beg \\ Aga Khan University
}

Follow this and additional works at: https://ecommons.aku.edu/pakistan_fhs_mc_pathol_microbiol

Part of the Diseases Commons, Health and Physical Education Commons, Microbiology Commons, and the Pathology Commons

\section{Recommended Citation}

Khan, Y. A., Mian, U. H., Ghanchi, N. K., Zubairi, A., Beg, M. A. (2018). Neurological involvement associated with plasmodium vivax malaria from Pakistan. Tropical Doctor, 48(1), 52-54.

Available at: https://ecommons.aku.edu/pakistan_fhs_mc_pathol_microbiol/723 


\section{Neurological involvement associated with Plasmodium vivax malaria from Pakistan}

Yousaf Abdullah Khan ${ }^{1, \S}$,Usman Hameed Mian ${ }^{1, \S}$, Najia Karim Ghanchi ${ }^{2}$, Ali Bin

Sarwar Zubairi ${ }^{3}$, Mohammad Asim Beg*,2

${ }^{\S}$ Equal contribution

${ }^{1}$ Medical college, Aga Khan University, Karachi, Pakistan

${ }^{2}$ Department of Pathology and Laboratory Medicine, Aga Khan University, Stadium Road, P.O. Box 3500, Karachi 74800, Pakistan.

${ }^{3}$ Department of Medicine, Aga Khan University, Stadium Road, P.O. Box 3500, Karachi 74800, Pakistan.

Short title: Neurological involvement associated with Plasmodium vivax Keywords: Cerebral malaria, Plasmodium vivax, gametocytes, seizures.

*Corresponding author: Mohammad Asim Beg masim.beg@aku.edu $\underline{\text { Tel\# } 009234864512 \text { / } 009234861641}$

\section{Background:}

Plasmodium vivax is the most common species causing malaria outside Africa with approximately 13.8 million reported cases worldwide. More than $80 \%$ of $P$. vivax malaria cases are estimated to occur in Pakistan, India and Indonesia (WHO 2015) . P.vivax is associated with increased morbidity but reduced mortality when compared to Plasmodium falciparum. Recently, studies from Indonesia, Papua New Guinea, Brazil, India, and Pakistan have shown that P.vivax, can independently prove fatal or result in severe morbidity. The most common complications of vivax malaria reported globally are anemia and non-cardiogenic pulmonary oedema/ acute respiratory distress syndrome. Other complications are severe thrombocytopenia, 
pancytopenia, jaundice, splenic rupture, acute renal failure and rarely cerebral involvement.

Cerebral malaria is a known complication of $P$. falciparum with over 575,000 cases leading to neurological, behavioural and cognitive defects reported annually.(1) However, cerebral malaria due to $P$. vivax mono infection is rarely reported. Malaria remains an endemic disease in Pakistan with an estimated health care burden of 1.6 million cases annually with $P$. vivax accounting for $67 \%$ of reported cases. We report a severe $P$. vivax infection with cerebral involvement in a child.

\section{Case Report}

A nine-year old boy presented to the emergency department of The Aga Khan University Hospital (AKUH) with a 3-day history of high grade fever accompanied by projectile vomiting and abnormal behaviour. A day prior to his admission he developed generalized tonic-clonic seizures associated with dizziness and unconsciousness. He had a coma score of $5 / 15$ on presentation with a tachycardia (167/min), tachypnoea (70 breaths/min), oxygen saturation of $92 \%$, blood pressure of $133 / 87 \mathrm{~mm}$ of $\mathrm{Hg}$ and a temperature of $38^{\circ} \mathrm{C}$. There was hypertonia in all limbs. The rest of the physical examination was normal. The full blood count showed anaemia ( $\mathrm{Hb} 94 \mathrm{~g} / \mathrm{L})$, a leucocytosis $\left(13.1 \times 10^{6} / \mathrm{mL}\right.$, neutrophils $75 \%$ and monocytes $8 \%$ ) and normal platelet count $\left(304 \times 10^{6} / \mathrm{mL}\right)$. A cerebral CT scan showed oedema with increased intracranial pressure. Thick and thin peripheral smear with Giemsa stain was suggestive of P. vivax with 1-10 per 100 high power fields. Stained blood films were also independently reviewed by an external expert parasitologist who confirmed the original diagnosis. Polymerase chain reaction (PCR) and an immunochromatography test (ICT) definitely excluded P. falciparum infection. Prothrombin time and activated partial thrombin time were in the normal range, and the direct antiglobulin test was negative. Although no lumbar puncture was performed empirical therapy for bacterial and viral meningitis was initiated. Initially Vancomycin, Meropenem, Acyclovir, steroids and anticonvulsants were started in emergency. The patient was mechanically ventilated. He required vasopressor support with norepinephrine. Blood cultures and urine cultures were all negative, Antibiotics were 
deescalated and stopped as blood film confirmed malarial parasite P.vivax and started with artemether lumefantrine. On the third day, he developed high urine output and hyponatremia secondary which was managed with fludrocortisone. On presentation his sodium levels were $145 \mathrm{mmol} / \mathrm{L}$ (normal ranges 135-145 mmol/L) and dropped to $125 \mathrm{mmol} / \mathrm{L}$ on third day. His sodium level was corrected to 135 in 72 hours and child gained consciousness. The GCS score improved to $15 / 15$ and he was extubated on the sixth day of admission and discharged on the ninth day.Post-treatment microscopy revealed blood films negative for P.vivax.

\section{Discussion}

$P$. vivax malaria has historically been termed, 'Benign Tertian Malaria' but it is increasingly felt that this is an erroneous label. Rossle in 1921 described a lethal cerebellar haemorrhage in a 21-year old soldier suffering a vivax infection

(3). Patients presenting with cerebral symptoms such as acute febrile encephalopathy, raised intracranial pressure, intracerebral haemorrhage, hemiparesis, aphasia and seizures following vivax malaria have been reported previously $(4,5)$.

Several cases of cerebral malaria attributed to P.vivax infection have been reported in recent years, especially in Asia and South America. This may be due to an overall increase in P. vivax malarial infections. Most of these cases have been reported from India where $P$. vivax is the predominant species (6). Although the mechanism by which P. vivax causes cerebral malaria is not fully understood, a suggested pathogenesis is the sequestration of infected erythrocytes in cerebral microvascular beds(7).

In our case, the peripheral blood smears with Giemsa stain were repeatedly performed and parasitaemia remained low. Immuno-chromatography testing showed no trace of $P$. falciparum, and PCR helped confirm mono-infection by $P$. vivax. Advances in molecular and serodiagnosis of malaria parasites have increased the sensitivity and specificity, and so the substantial accuracy, of diagnosis of P. vivax. $(8,9)$ Physicians must be aware of the aggressive pathology of severe P. vivax infections and institute prompt and accurate diagnosis in treatment. Furthermore, an increasing 
incidence of severe malaria cases due to P. vivax are already placing a huge burden on an already strained healthcare system.

The study was approved by the AKUH ethical review committee (reference no. 3541Path-ERC-15). Informed consent was obtained from the patient for the publication of this report.

The authors declare that they have no competing interests, and received no funding.

Author contributions: MAB, SAZ, YAK, MUH were responsible for clinical work, designed and planned the study. NKG performed Microscopy, PCR genotyping and interpretation. AAW and YAK, MUH, NKG prepared the initial manuscript. MAB and SAZ reviewed the final draft. All authors read and approved the final manuscript.

\section{References}

1. Idro R, Marsh K, John CC, Newton CRJ. Cerebral Malaria; Mechanisms Of Brain Injury And Strategies For Improved Neuro-Cognitive Outcome. Pediatric research. 2010;68(4):267-74.

2. Padley D, Moody AH, Chiodini PL, Saldanha J. Use of a rapid, single-round, multiplex PCR to detect malarial parasites and identify the species present. Ann Trop Med Parasitol. 2003;97(2):131-7.

3. Vinken P, Bruyn G. Handbook of Clinical Neurology: North Holland; 1969.

4. Rathia SK, Sankar J, Kandasamy D, Lodha R. Plasmodium vivax Malaria Presenting with Multifocal Hemorrhagic Brain Infarcts in a School-going Child. J Trop Pediatr. 2016.

5. Karanth SS, Marupudi KC, Gupta A. Intracerebral bleed, right haemiparesis and seizures: an atypical presentation of vivax malaria. BMJ Case Rep. 2014;2014.

6. Kochar DK, Das A, Kochar SK, et al. Severe Plasmodium vivax malaria: a report on serial cases from Bikaner in northwestern India. Am J Trop Med Hyg. 2009;80(2):194-8.

7. Wassmer SC, Combes V, Grau GE. Pathophysiology of cerebral malaria: role of host cells in the modulation of cytoadhesion. Ann N Y Acad Sci. 2003;992:30-8 
8. Abba K, Kirkham AJ, Olliaro PL, et al. Rapid diagnostic tests for diagnosing uncomplicated non-falciparum or Plasmodium vivax malaria in endemic countries. Cochrane Database Syst Rev. 2014;12:11431.

9. Valecha N BA, Chandra J, Sharma D. Cerebral symptoms with P. vivax malaria. . Indian Pediatr 1992;29: 1176-8. 